\title{
Attempts at the development of a recombinant African swine fever virus strain with abrogated EP402R, 9GL, and $A 238 L$ gene structure using the CRISPR/Cas9 system
}

\author{
Grzegorz Woźniakowski ${ }^{1}$, Natalia Mazur-Panasiuk ${ }^{1 凶}$, Marek Walczak ${ }^{1}$, \\ Małgorzata Juszkiewicz ${ }^{1}$, Maciej Frant ${ }^{1}$, Krzysztof Niemczuk ${ }^{2}$ \\ ${ }^{1}$ Department of Swine Diseases, ${ }^{2}$ Director General \\ National Veterinary Research Institute, 24-100 Puławy, Poland \\ natalia.mazur@piwet.pulawy.pl
}

Received: October 4, 2019 Accepted: May 25, 2020

\begin{abstract}
Introduction: African swine fever (ASF) is a pressing economic problem in a number of Eastern European countries. It has also depleted the Chinese sow population by $50 \%$. Managing the disease relies on culling infected pigs or hunting wild boars as sanitary zone creation. The constraints on the development of an efficient vaccine are mainly the virus' mechanisms of host immune response evasion. The study aimed to adapt a field ASFV strain to established cell lines and to construct recombinant African swine fever virus (ASFV) strain. Material and Methods: The host immune response modulation genes A238L, EP402R, and $9 G L$ were deleted using the clustered regularly interspaced short palindromic repeats/caspase 9 (CRISPR/Cas9) mutagenesis system. A representative virus isolate (Pol18/28298/Out111) from Poland was isolated in porcine primary pulmonary alveolar macrophage (PPAM) cells. Adaptation of the virus to a few established cell lines was attempted. The plasmids encoding CRISPR/Cas9 genes along with gRNA complementary to the target sequences were designed, synthesised, and transfected into ASFV-infected PPAM cells. Results: The reconstituted virus showed similar kinetics of replication in comparison to the parent virus isolate. Conclusion: Taking into account the usefulness of the developed CRISPR/Cas9 system it has been shown that modification of the $A 238 \mathrm{~L}, E P 402 R$, and $9 G L$ genes might occur with low frequency, resulting in difficulties in separation of various virus populations.
\end{abstract}

Keywords: African swine fever, CRISPR/Cas9, EP402R, 9GL, A238L.

\section{Introduction}

African swine fever (ASF) was introduced to Poland in February 2014 most probably via a dead wild boar $(27,32,34)$. In spite of efforts made to manage the population of wild boars and thus reduce the potential rate of African swine fever virus (ASFV) transmission between infected and susceptible animals, the disease is continuously spreading to new areas. Recently, numerous wild boar cases were reported for the first time in Lubuskie province in western Poland, about $300 \mathrm{~km}$ away from the nearest previously reported cases in Masovian province. This unexpected turn caused severe concern about further spread of the disease into Greater Poland province, where the pig population has high density, and also to neighbouring Germany. As a consequence of ASF prevalence among infected wild boars, the disease outbreaks in domestic pigs are closely aligned to the same areas where infected boars are found. Up to the end of February 2020 in Poland, the total number of ASF cases in wild boars had reached nearly 7,000, while in domestic pigs, 261 outbreaks had been notified. While ASF is not infectious to humans, it substantially impacts the national and international agriculture sector economically and socially (8). Because of the lack of an effective vaccine, the only control strategy stipulates two drastic measures: one, ruinous to pig producers, of 
culling livestock and the other, of hunting campaigns in order to reduce wild boar populations to the minimum possible level of $0.1-0.5$ animals per square kilometre $(9,14)$. The only reasonable means of ASF prevention is implementation of high level biosecurity practices in every kind of pig holding (15). Meanwhile, taking into account that currently the seventh wave of ASF is inflicting mortalities on domestic pigs in Poland, the potential risk of ASF occurrence is also real in commercial farrow-to-finish or fattening farms of up to 30,000 head.

ASF was originally described by Montgomery in 1921 in Kenya, but since then it has been present in many countries of Europe, South America, and recently also in Asia. Therefore, it has ceased to be considered an exotic disease $(5,9)$. ASFV is a large multienveloped DNA virus and the sole member of the Asfivirus genus in the Asfarviridae family (7). Its complex genome comprises a single, linear doublestranded DNA molecule, encoding genes essential in viral replication, virus assembly, and egress as well as responsible for immunological interactions with the host (7). The virus replicates predominantly in cells belonging to the mononuclear phagocyte system, specifically monocytes and macrophages, but nevertheless, other cell types may also be infected in the late stages of infection (6). ASFV possesses a unique feature to avoid recognition by and cytotoxic response of $\mathrm{CD} 8+$ cells $(26,33)$. Neutralising antibodies are not produced as they are in the in case of a number of other viral pathogens of pigs. Several viral genes which block or manipulate the host immune response have been identified (7). Among them, the A238L gene has an important role in the inhibition of expression of transcription factors involved in host immune response (29). Another gene, EP402R encoding CD2-like protein, is responsible for adsorption of erythrocytes around infected cells (haemadsorption) and facilitates virus spread in the host (7). Deletion or alteration of the EP402R gene in virulent ASFV strains leads to haemadsorption abrogation in vitro, furthermore in animal trials, it led to delayed viraemia and clinical signs, suggesting the crucial role of this gene in ASFV virulence $(1-3,11$, 13, 21). Similarly, the studies conducted on ASFV strain lacking the $9 G L$ gene showed this gene's important role in maturation of fully infectious ASFV particles. Moreover, in vivo study demonstrated that the strain with deleted $9 G L$ was attenuated in pig model $(19,24)$.

The recent ASFV pandemic has highlighted the urgent need for vaccine development. Inactivated or subunit vaccines showed no protective immunity (12, 22 ), while some naturally attenuated strains isolated from the field are able to provide complete protection against virulent strains $(2,35)$. Partial or full protection was induced by live attenuated vaccines (LAVs) obtained by serial passages in cell cultures or targeted deletion of selected genes $(16,23-25)$. LAVs present the most promising candidates; nevertheless, further studies are required to guarantee their safety, long-term efficacy, and capacity to differentiate between infected and vaccinated animals. Investigations regarding ASFV vaccine are continuously impeded by deficient knowledge about virus interactions with host immunity, its genetic complexity, and technical difficulties such as the lack of established ASFV-permissive cell lines or low transfection efficiency in porcine macrophages (8).

During the last few years, CRISPR/Cas9 (clustered regularly interspaced short palindromic repeat/caspase 9) technology has become an exceptionally efficient method of genetic manipulation of distinct eukaryotic and prokaryotic organisms; moreover, this powerful tool facilitated targeted viral gene modifications (18). Originally, CRISPR/Cas9 was described as a component of the $S$. pyogenes immune system, which allows bacteria to combat phages in a simple but precise manner engaging endonuclease and RNA (17). In gene engineering applications, binding of Cas9 endonuclease to the target site is mediated by about 20-nt-long guide RNA (gRNA) complementary to the target sequence and a 3-nt-long protospacer adjacent motif (PAM) located immediately downstream of a given locus. Cas9 protein induces a double-strand break (DSB) within the target, about 3 or 4 nt upstream of the PAM sequence. Subsequently, the DSB is repaired by the nonhomologous end joining (NHEJ) pathway, resulting in minor nucleotide insertions or deletions and therefore in disruption of the encoded gene open reading frame (ORF) (17).

ASFV replication was successfully inhibited by this RNA-directed DNA modification technique targeting the p30 gene (13). This technology seems to be a promising approach to generation of a live attenuated ASFV vaccine strain. The study presented in this paper aimed to adapt an ASFV field isolate to established cell lines and to perform ASFV genomic manipulation in order to develop a recombinant strain lacking three immune response inhibition-related genes (9GL, A238L, and EP402R).

\section{Material and Methods}

Cell cultures and viruses. Porcine primary pulmonary alveolar macrophages (PPAMs) were purchased from the Technical University of Denmark (DTU, Lindholm, Denmark), primary cultures of pig bone marrow (PBM) cells were obtained by washing out the red bone marrow derived from the long bones of $20 \mathrm{~kg}$ Polish Large White pigs. Immortalised porcine alveolar macrophage (IPAM) and MARC-145, COS-1, and Vero cell lines were purchased from ATCC. PPAM, PBM, and IPAM cells were subcultured in RPMI 1640, 10\% FBS, 1\% antibiotic-antimycotic solution, $1 \mathrm{mM}$ sodium pyruvate, and $1 \times$ non-essential amino acids. MARC-145 and COS-1 were maintained 
in Dulbecco's modified Eagle medium (DMEM) and Vero in MEM, all supplemented with $10 \%$ FBS and 1\% antibiotic-antimycotic solution. All cultures were grown at $37^{\circ} \mathrm{C}$ in a humidified atmosphere containing $5 \% \mathrm{CO}_{2}$.

The ASFV Pol18/28298/Out111 genotype II isolate was derived from the spleen of a pig which died during outbreak 111, (Chełm district, Poland, May 2018). Virus was isolated from clinical material as previously described (20). The titre was calculated using the Reed-Muench method (28).

The growth kinetics of isolated virus were evaluated in PPAM cells, which were infected with ASFV Pol18/28298/Out111 at a multiplicity of infection (MOI) of 0.1 . Subsequently medium samples collected $0,1,2,3$, and 4 days post infection (dpi) were titrated.

Assessment of susceptibility of selected established cell lines to ASFV infection. Since field isolates of ASFV belonging to genotype II are not capable of infecting established cell lines, we attempted to adapt Polish field strain of ASFV to continuous cell lines, which were previously shown to facilitate replication of other ASFV genotypes. Nevertheless, up to date, only one paper reported successful adaptation of ASFV genotype II strain Georgia 2007/1 to such a culture (16).

In order to perform adaptation, subconfluent monolayers of the IPAM, MARC-145, COS-1, and Vero cell lines were infected either with spleen homogenate or virus stock previously isolated in PPAM cells. The infected cultures were then incubated for five days, and subsequently, 10 passages were performed. A real-time PCR according to FernandezPinero (10) was used to control virus replication. At 5 dpi of the tenth passage, cells were fixed, and an immunoperoxidase test (IPT) was carried out in order to confirm or rule out the presence of infected cells, since neither haemadsorption nor cytopathic effect was observed.

Construction of CRISPR/Cas9 expression plasmids. In total, six DNA fragments encoding gRNA which aligns to six selected regions within the $9 G L$, $A 238 L$, and EP402R ASFV genes (two target sites per gene) were designed (Table 1). Target sequences were unique in comparison to the rest of genome and were located adjacent to a 3-nt-long PAM sequence serving as a binding signal for Cas9 nuclease. The pGK1.1 vector was obtained by cloning the designed gRNA encoding oligonucleotides into a vector backbone (Creative Biogene, Shirley, NY, USA). Target sequences including PAM and their positions are presented in Table 1.

The plasmid encodes an endonuclease Cas9 (Csn1) and two antibiotic resistance genes (kanamycin and puromycin) to facilitate selection of positive clones. The sequence encoding gRNA and CRISPR RNA is encoded under the control of a U6 promoter.
E. coli G10 cells were transformed with the obtained construct and provided by Creative Biogene as bacterial glycerol stocks. E.coli were cultivated overnight in LB medium supplemented with kanamycin, then sedimented by centrifugation, and subjected to plasmid DNA extraction with a NucleoSpin Plasmid Miniprep Kit (Machery Nagel, Hoerdt, Germany) according to the manufacturer's protocol. The correctness of the obtained plasmids was verified by a conventional PCR utilising a forward primer aligning to the right end of the U6 promoter (CATATGCTTACCGTAACTTGAAAG) and reverse complement target sequences as the reverse primer. The annealing temperature was set at $50^{\circ} \mathrm{C}$. The plasmids were used for further transfection of selected cell lines.

Transfection. Six plasmids containing the CRISPR/Cas 9 encoding cassette, specific for six target sequences (two vectors per gene, each of which targeted two distinct sequences), were designed for transfection. During this study, two transfection kits were tested, namely the Xfect (TaKaRa Bio, Mountain View, CA, USA) and the GeneJect transfection reagents (A\&A Biotechnology, Gdynia, Poland). The former is a biodegradable transfection polymer stated by the producer to have a very low cytotoxicity profile and high transfection efficiency (up to 97\%). The latter has reduced cell toxicity compared to lipofectin; however, its toxicity may increase at low confluence. A comparison of the protocols for the transfection kits used during this study is shown in Table 2. The Xfect and GeneJect transfection reagents were used for transfection of primary cells (PPAM) and a continuous cell line (Vero), and the outcomes informed the selection of the appropriate transfection kit and target cells, which is schematised in Fig. 1. The transfected cells were intended for further virus infection.

Infection. Infection was carried out in 24-well plates with $1 \times 10^{6}$ cells $/ \mathrm{mL}$ and an MOI of 0.1 . After 48-72 h, the haemadsorption phenomenon was noticeable, and at $7 \mathrm{dpi}, 100 \mu \mathrm{L}$ of thrice freeze-thawed medium was transferred to fresh PPAM cell cultures to observe replication of the virus.

Transfected cells (PPAMs and primary PBM cells) were subjected to 24 - or 48-h puromycin selection, and subsequently infected with ASFV Pol18/28298/Out111 in the presence of pig erythrocytes in order to observe haemadsorption. At $5 \mathrm{dpi}$, the plates were freezethawed three times, the total DNA was extracted, and ASFV presence was confirmed by real-time PCR (10). The targeted $9 G L, A 238 L$, and EP402R genes of the ASFV genome were amplified by conventional PCR and 1.4-, 0.759-, and 1,139-kbp regions covering the whole genes of interest were sequenced. Moreover, amplification of the $A 224 L$ gene was performed as a reaction control. The primer characteristics are given in Table 3. 
Table 1. Target DNA sequences of $A 238 L, E P 402 R$, and $9 G L$ genes of African swine fever strains. Nucleotide positions refer to the ASFV Georgia 2007/1 genome sequence (GenBank accession number FR682468.1). PAM sequences are marked in bold

\begin{tabular}{llll}
\hline Name & Sequence & Position & Length \\
\hline A238L-1 & CCGAAATAGCCCAACACCCCTTC & $50.832-50.851$ & 20 \\
A238L-2 & TCGCATCTATTGACAATCCACGG & $51.027-51.046$ & 20 \\
EP402R-1 & CCTCATAATGATGTATTTGATAC & $73.627-73.646$ & 20 \\
EP402R-2 & CCTGCTACTCCCCCAAATATCAC & $73.705-73.724$ & 20 \\
9GL-1 & CCAGTACTGAAAGTCCTCCGAG & $95.099-95.117$ & 19 \\
9GL-2 & CCAGTATTTAGGTCCCCAATGCA & $95.285-95.304$ & 20 \\
\hline
\end{tabular}

Table 2. Transfection protocol. The main conditions for two applied kits are presented

\begin{tabular}{lll}
\hline Parameter & Xfect & GeneJect \\
\hline Plasmid DNA & $5 \mu \mathrm{g}$ & $0.4 \mu \mathrm{g}$ \\
Total growth medium volume & $1,000 \mu \mathrm{L}$ & $1,000 \mu \mathrm{L}$ \\
Transfection reagent & $1.5 \mu \mathrm{L}$ & $2 \mu \mathrm{L}$ \\
Incubation time for nanocomplexes creation & $10 \mathrm{~min} / \mathrm{RT}$ & $30 \mathrm{~min} / \mathrm{RT}$ \\
Incubation time with target cells & $4 \mathrm{~h} / 37^{\circ} \mathrm{C}$ & $24-72 \mathrm{~h} / 37^{\circ} \mathrm{C}$ \\
Additional steps & Disposal of medium & - \\
Control of transfection effect & Replacement with fresh growth medium & Within $24-72 \mathrm{~h}$ incubation time \\
\hline
\end{tabular}

$\mathrm{RT}$ - room temperature

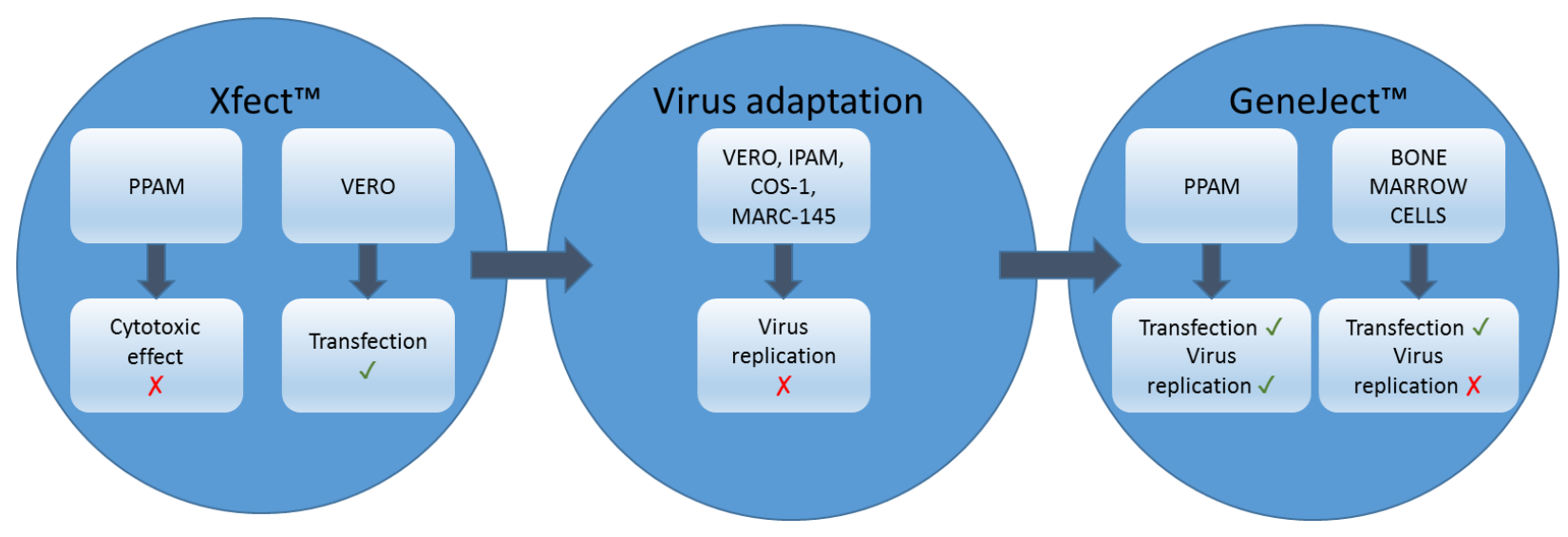

Fig. 1. Schematic representation of the selection of the suitable transfection kit and target cells

Table 3. Primers used for amplification of the region of interest covering target genes. Nucleotide positions refer to the ASFV Georgia 2007/1 genome sequence (GenBank accession number: FR682468.1)

\begin{tabular}{llll}
\hline Name & Sequence & Position & $\begin{array}{l}\text { Tm (Primer melting } \\
\text { temperature) }\end{array}$ \\
\hline A238L-F & TTGGACACAGGAAACGATCT & $50.370-50.389$ & $49.7^{\circ} \mathrm{C}$ \\
A238L-R & ATATGGGAAAAGGGCCTGGC & $51.302-51.283$ & $53.8^{\circ} \mathrm{C}$ \\
EP402R-F & ACTATATTATAAAACATATG & $73.341-73.360$ & $37.4^{\circ} \mathrm{C}^{\circ}$ \\
EP402R-R & TGCATGTGATGGAAATCGGT & $74.594-74.575$ & $49.7^{\circ} \mathrm{C}$ \\
9GL-F & GCCTCACTATCGATCGGCAA & $94.046-94.065$ & $53.8^{\circ} \mathrm{C}$ \\
9GL-R & ACTGGCTGGAATTACGCCAA & $95.450-95.431$ & $51.8^{\circ} \mathrm{C}$ \\
A224L-F & AAAAGCTATTTGTTTATCCCCA & $46.266-46.287$ & $47.4^{\circ} \mathrm{C}$ \\
A224L-R & CCTTCAATTGAGGATGATCATT & $47.057-47.036$ & $49.2^{\circ} \mathrm{C}$ \\
\hline
\end{tabular}




\section{Results}

Field ASFV strain. ASFV Pol18/28298/Out111 isolated in PPAM cells reached a titre of $6.06 \log _{10} \mathrm{HAD}_{50} / \mathrm{mL}$. The haemadsorption phenomenon was easily observable from $1 \mathrm{dpi}$ and was characteristic for the ASFV genotype II, currently circulating in Europe and Asia. The replication kinetics assessed in PPAM were comparable to previously reported growth kinetics of the reference Georgia 2007/1 strain (23). Sanger sequencing of the three target genes showed $100 \%$ identity with this strain.

Adaptation to established cell lines. Unfortunately, despite strenuous attempts at the development of a strain adapted to established cell lines, each of the tested cells (IPAM, MARC-145, COS-1, and Vero) was incapable of facilitating and maintaining ASFV replication during ten passages. Neither cytopathic effect nor haemadsorption phenomenon were visible; moreover, real-time PCR results clearly confirmed that the ASFV Pol18/28298/Out111 strain (both directly from clinical samples and previously isolated in PPAMs) is not able to grow in the selected continuous cell lines because viral DNA content fell successively, disappearing completely between the second and eighth passages. The IPT assay performed during the tenth passage showed the absence of replicating virus. This result is in line with previous reports that ASFV genotype II strains replicate in primary cells belonging to the mononuclear phagocyte system (6) rather than in continuous cells.
Vector design. In order to confirm the correctness of the extracted plasmids, a conventional PCR amplifying the region between the $3^{\prime}$ end of the U6 promoter and the target sequence was performed. The obtained products were about $100 \mathrm{bp}$ in size, which was consistent with expectations and evidenced the presence of the gRNA encoding sequence within the vector backbone.

Transfection. As regards transfection, the X-fect reagent yielded successful results exclusively in an established cell line (Vero), which was able to grow stably in medium supplemented with $1 \mu \mathrm{g} / \mathrm{mL}$ of puromycin (Fig. 2).

Nevertheless, these transfection reagents transpired to be cytotoxic to PPAM cells, and when used according to the manufacturer's protocol, caused cell death almost immediately. For this reason, the GeneJect transfection kit dedicated to more susceptible and hard-to-transfect cells was used with PPAM and PBM cells and exerted no cytotoxic effect. It is apposite to confirm that transfection of each of the six plasmids was conducted separately in order to determine whether any of the induced mutations directly affected virus replication.

Infection. Puromycin-resistant PPAMs and PBM cells were infected with ASFV Pol18/28298/Out111, 24 or $48 \mathrm{~h}$ post transfection, and the virus was allowed to propagate for seven days, during which haemadsorption was observed. Subsequently, a realtime PCR was performed. The obtained results are included in Table 4 and clearly show that virus replication is present in PPAM cells.
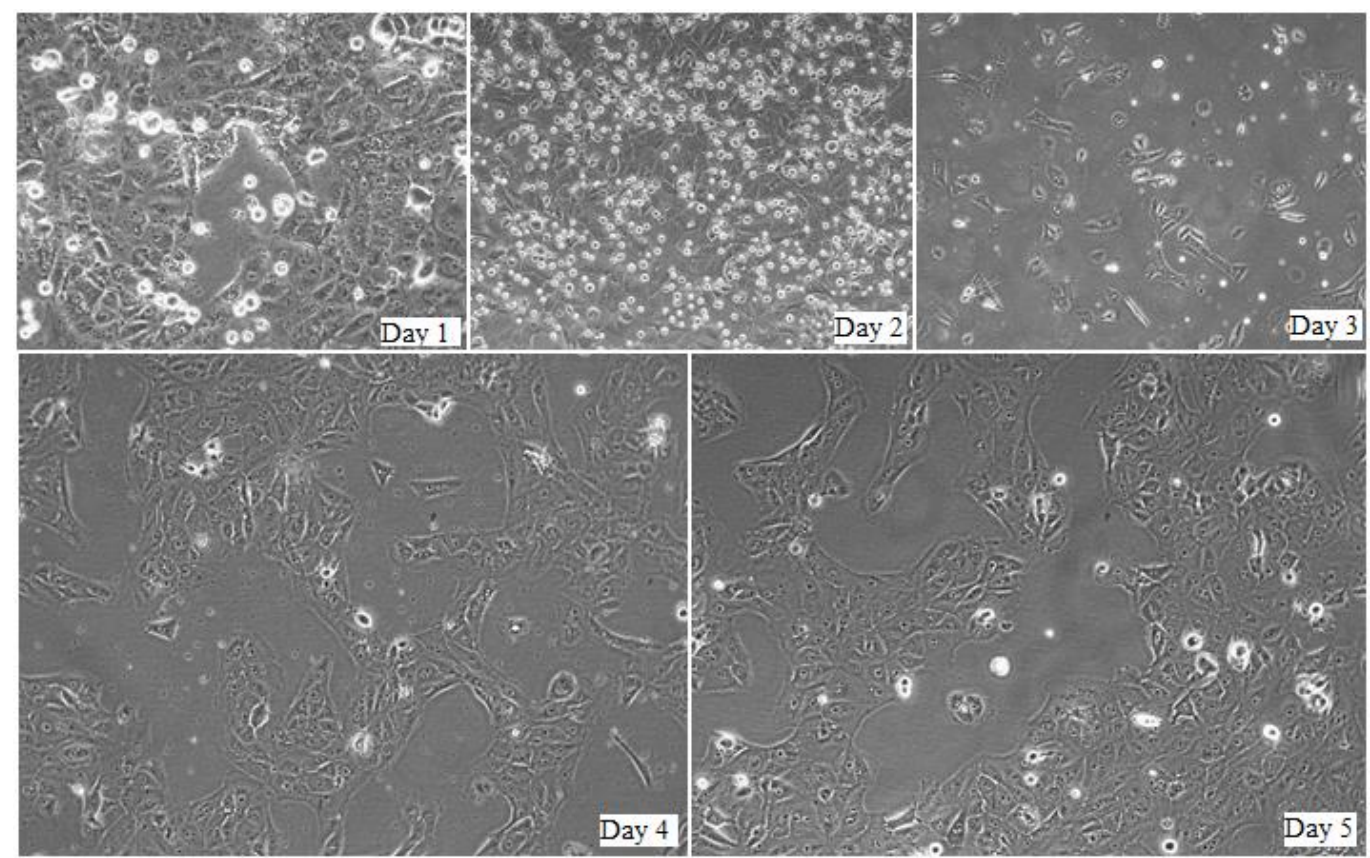

Fig. 2. Vero cells transfected with constructed CRISPR/Cas9 plasmid. In order to confirm successful transfection, a puromycin selection was applied, which led to an increased rate of cell death during the first three days post transfection, and later the cell culture started to grow in antibiotic supplemented medium (magnification $200 \times$ ) 
Target gene analysis. The viruses obtained in the second passage after $48 \mathrm{~h}$ incubation with puromycin were selected for further analysis. A conventional PCR was performed in order to amplify viral target genes as well as the $A 224 L$ gene which was used as the PCR control for each of the obtained knock-out (KO) viruses (Fig. 3). Lack of $A 224 L$ amplification in KO-9GL1 and KO-EP402R2 was probably a consequence of the low ASFV DNA concentration (35.97 and $36.25 \mathrm{Cq}$ values in qPCR, respectively). Therefore, only KO-9GL2 and KO-EP402R1 were analysed further. The results showed no PCR product of the $9 G L$ and EP402R sequences for cells transfected with each of the plasmid variants (Table 5). However, the cells transfected with the A238L-1 and A238L-2 target sequence-encoding plasmids showed appropriately sized bands; therefore, the PCR product was subjected to further sequencing. The findings revealed the $100 \%$ nucleotide identity of the obtained recombinant viruses with the parent ASFV Pol18/28298/Out111.

Replication kinetics. The in vitro replication kinetics of each of the six generated deletion mutants and WT-ASFV/Pol18/28298/O111 were evaluated using a culture of primary swine macrophages. The cells were infected with the analysed viruses at an MOI of 0.1 , and samples were collected at $0,1,2,3$, and 4 dpi. All evaluated strains showed similar growth kinetics to each other and their parent strain (Fig. 4).

Table 4. Results obtained in real-time PCR

\begin{tabular}{|c|c|c|c|c|c|c|c|c|}
\hline \multirow{2}{*}{ Target site } & \multicolumn{2}{|c|}{ PPAM Puromycin $24 \mathrm{~h}$} & \multicolumn{2}{|c|}{ PPAM-Puromycin $48 \mathrm{~h}$} & \multicolumn{2}{|c|}{ PBM Puromycin 24 h } & \multicolumn{2}{|c|}{ PBM Puromycin $48 \mathrm{~h}$} \\
\hline & I & II & I & II & I & II & I & II \\
\hline 9GL-1 & $33.89^{\mathrm{h}}$ & $33.85^{\mathrm{h}}$ & $34.14^{\mathrm{h}}$ & 35.97 & $31.95^{\mathrm{h}}$ & - & $31.48^{\mathrm{h}}$ & 38.62 \\
\hline 9GL-2 & $34.49^{\mathrm{h}}$ & $31.05^{\mathrm{h}}$ & $34.38^{\mathrm{h}}$ & $31.99^{\mathrm{h}}$ & $31.65^{\mathrm{h}}$ & $33.55^{\mathrm{h}}$ & $32.37^{\mathrm{h}}$ & 34.08 \\
\hline A238L-1 & $32.26^{\mathrm{h}}$ & $31.3^{\mathrm{h}}$ & $32.75^{\mathrm{h}}$ & $31.26^{\mathrm{h}}$ & $32.34^{\mathrm{h}}$ & $28.5^{\mathrm{h}}$ & $32.88^{\mathrm{h}}$ & - \\
\hline A238L-2 & $32.92^{\mathrm{h}}$ & $31.93^{\mathrm{h}}$ & $32.92^{\mathrm{h}}$ & $29.88^{\mathrm{h}}$ & $31.93^{\mathrm{h}}$ & $30.52^{\mathrm{h}}$ & $33.61^{\mathrm{h}}$ & 35.54 \\
\hline EP402R-1 & $31.92^{\mathrm{h}}$ & 38.71 & $33^{\mathrm{h}}$ & $29.76^{\mathrm{h}}$ & $32.74^{\mathrm{h}}$ & 34.21 & $32.9^{h}$ & $33.79^{h}$ \\
\hline EP402R-2 & $31.79^{\mathrm{h}}$ & $28.82^{\mathrm{h}}$ & $32.8^{\mathrm{h}}$ & 36.25 & $32.89^{\mathrm{h}}$ & 38.69 & $32.59^{\mathrm{h}}$ & 34.81 \\
\hline
\end{tabular}

${ }^{\mathrm{h}}$ - haemadsorption; I (II) - first (second) passage after 24 (48) h of incubation with puromycin

Table 5. Results of conventional PCR

\begin{tabular}{lllll}
\hline Material & PCR target & & & \\
\hline & $9 G L$ & $A 238 L$ & $E P 402 R$ & $A 224 L$ \\
KO-9GL1 & $(2)^{-}$ & $\mathrm{n} / \mathrm{a}$ & $\mathrm{n} / \mathrm{a}$ & $(5)-$ \\
KO-9GL2 & $(3)^{-}$ & $\mathrm{n} / \mathrm{a}$ & $\mathrm{n} / \mathrm{a}$ & $(6)+$ \\
KO-A238L1 & $\mathrm{n} / \mathrm{a}$ & $(8)+$ & $\mathrm{n} / \mathrm{a}$ & $(11)+$ \\
KO-A238L2 & $\mathrm{n} / \mathrm{a}$ & $(9)+$ & $\mathrm{n} / \mathrm{a}$ & $(12)+$ \\
KO-EP402R1 & $\mathrm{n} / \mathrm{a}$ & $\mathrm{n} / \mathrm{a}$ & $(14)-$ & $(17)+$ \\
KO-EP402R2 & $\mathrm{n} / \mathrm{a}$ & $\mathrm{n} / \mathrm{a}$ & $(15)-$ & $(18)-$ \\
WT & $(4)+$ & $(10)+$ & $(16)+$ & $(7,13,19)+$ \\
\hline
\end{tabular}

Numbers in brackets represent sample numbers in Fig. 3. KO - knock-out targets; WT - wild type; $\mathrm{n} / \mathrm{a}$ - not applicable

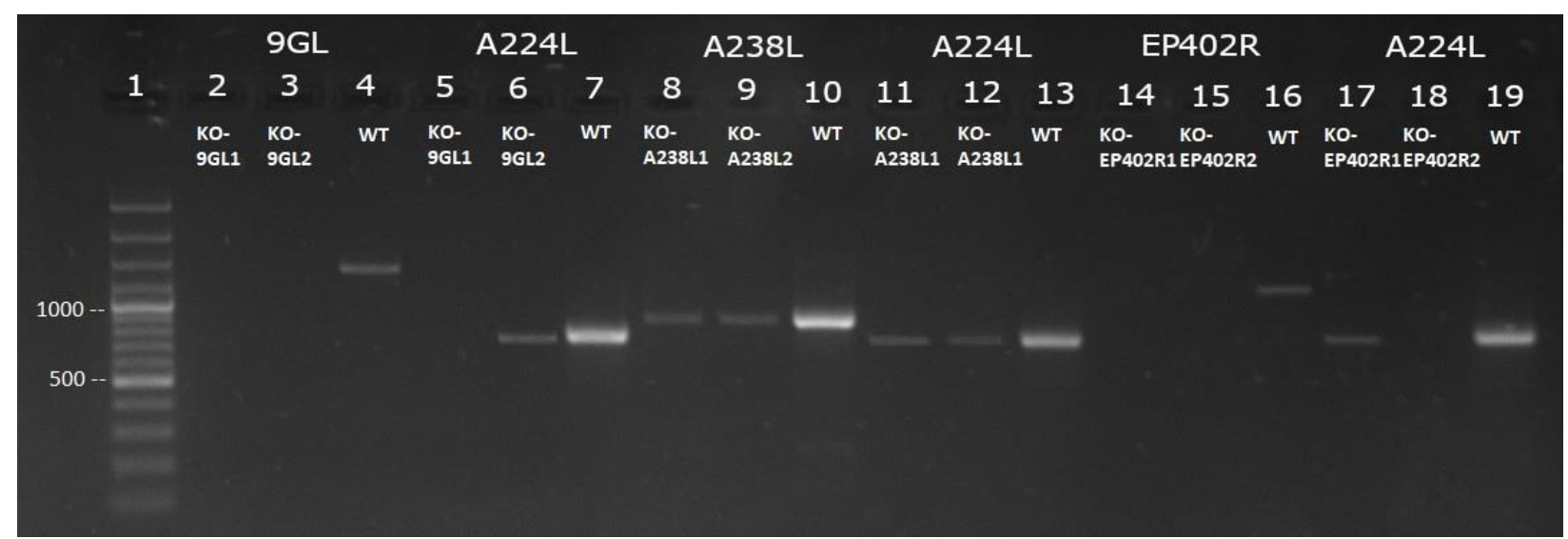

Fig. 3. Agarose gel (2\%) showing the results of the conventional PCR to amplify whole sequences of targeted genes. Gene names at the top represent the amplified region. Numbers below gene names correspond to Table 5 numbers in brackets, 1000/500 - band size markers 

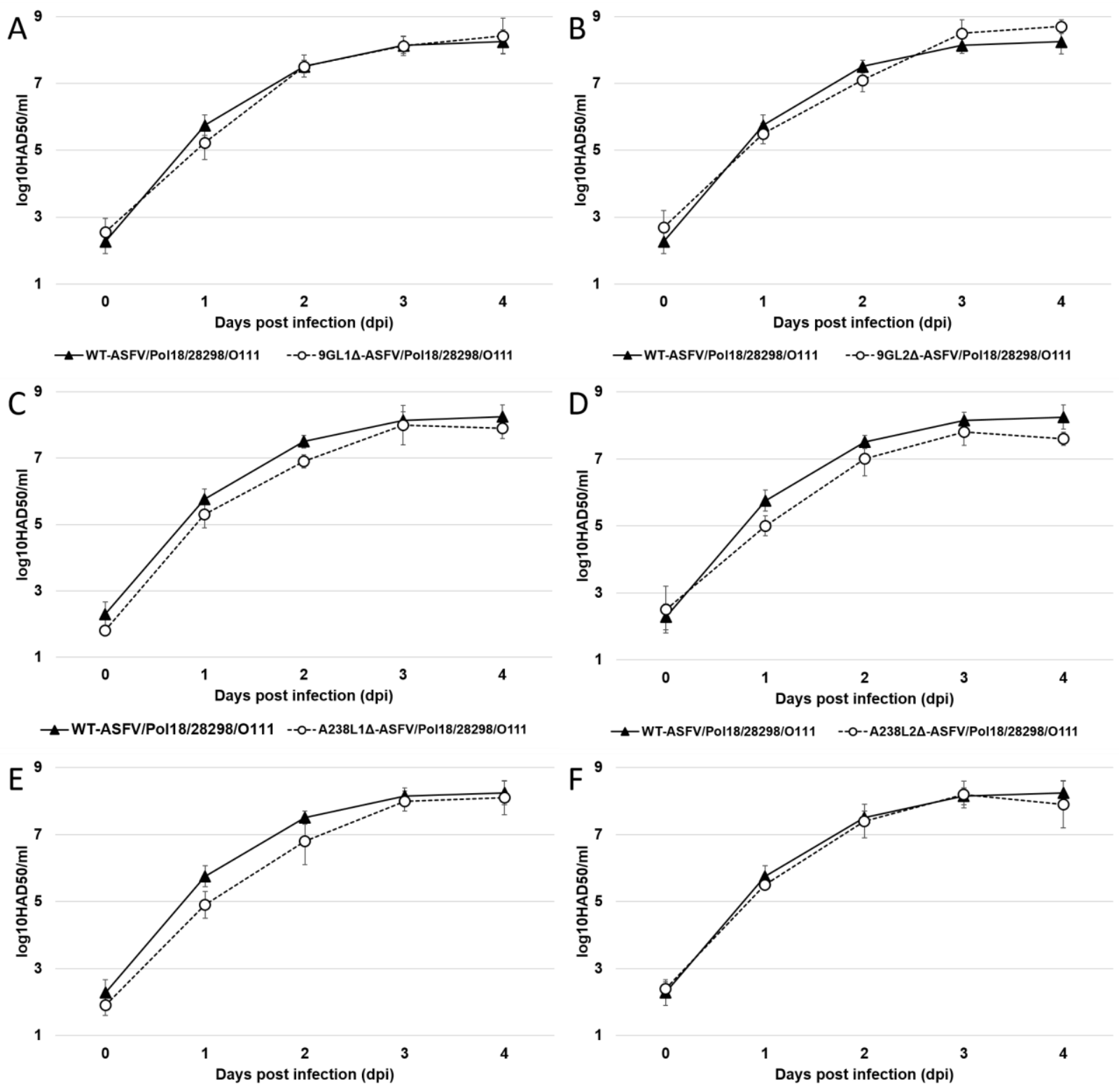

¿-WT-ASFV/Pol18/28298/0111 --O-EP402R1A-ASFV/Pol18/28298/0111

—-WT-ASFV/Pol18/28298/0111 --O-EP402R2A-ASFV/Pol18/28298/0111

Fig. 4. In vitro replication kinetics of the six generated deletion mutant (A - 9GL1 $\Delta$; B $-9 \mathrm{GL} 2 \Delta ; \mathrm{C}-\mathrm{A} 238 \mathrm{~L} 1 \Delta ; \mathrm{D}-\mathrm{A} 238 \mathrm{~L} 2 \Delta ; \mathrm{E}-\mathrm{EP} 402 \mathrm{R} 1 \Delta$; $\mathrm{F}-\mathrm{EP} 402 \mathrm{R} 2 \Delta$ ) and parent ASFV/Pol18/28298/O111 isolates. PPAM cell cultures were infected (MOI 0.1) with both strains, and subsequently virus titres were estimated daily over $96 \mathrm{~h}$ post infection. Data represent means and standard deviations from three independent experiments. The sensitivity of virus detection was $1.8 \mathrm{HAD}_{50} / \mathrm{mL}$

\section{Discussion}

To date, numerous research efforts have been made to develop a vaccine against ASFV; nevertheless, the only currently available methods to control the disease are based on early detection and implementation of strict biosecurity measures. However, these have proved to be insufficient to limit worldwide ASF spread; therefore, the generation of a safe, effective vaccine is urgently required. So far, attempts at its development have included inactivated viruses, recombinant proteins, DNAs-, and LAVsvaccines, but only the last approach seems to be a promising strategy to generate protective immunity in pigs and wild boars (30). LAVs which showed high levels of protection were obtained by serial passaging in cell cultures or by rational genetic modification of virulent strains. Moreover, naturally attenuated, low virulent $\mathrm{ASFV}$ isolates present a promising alternative to developing protection against lethal challenge with ASF virus homologues (2). The CRISPR/Cas9 technique has so far shown itself to be an effective approach for manipulation of the ASFV genome, efficiently inhibiting virus replication in vitro (13). Nevertheless, since highly virulent ASFV strains are only capable of infecting and efficiently replicating in primary cells (31), attempts to develop a genetically engineered virus are even more difficult.

Despite our best efforts to adapt Polish ASFV strains to numerous established cell lines, neither serial 
passages nor modifications of the medium composition in order to sensitise cells facilitated efficient virus replication in them. Transfection of continuous cell lines (Vero) was easier to conduct, observe, and control than transfection of primary cells; therefore, such an approach might be much more suitable for virus genetic manipulation purposes due to the constant process of cell proliferation. However, since established cell lines are not permissive of ASFV genotype II replication, and numerous attempts to adapt the virus have failed, the possibility of using stable cell lines in further studies must be discounted. As primary cells were found to be highly sensitive to transfection reagents, genetic manipulation of ASFV genotype II using plasmid-delivered modification systems such as CRISPR/Cas9 in combination with PPAM cell culture is severely constricted. Moreover, the lack of a gene encoding a fluorescent protein such as green or red (GFP or RFP) within the vector backbone caused the selection of positive transfected clones to be based only on antibiotic resistance genes. Such selection is not an optimal approach for primary, non-dividing cells; therefore, determination of the successfully transfected cell proportion is difficult, and may lead to replication of WT virus. Lack of amplification of the $9 G L$ and EP402R genes in the conventional PCR precluded unarguable confirmation of changes in its nucleotide sequences. However, similar growth kinetics of the altered and parent strains and the observation of a haemadsorption phenomenon in a strain intended not to include the $E P 402 R$ gene may respectively suggest that only a small proportion of viruses underwent successful modification of the $9 G L$ and $E P 402 R$ genes. As regards the $A 238 L$ gene, sequencing explicitly excluded any changes in its nucleotide sequence.

There are few possible explanations of the obtained results, two of which being that Cas9 nuclease has a low or limited affinity to the selected target sites and that deletions or insertions induced by the NHEJ pathway repair do not significantly affect protein function. Although CRISPR/Cas9 is considered a method which revolutionised genetic engineering, using this RNA-guided genome modification system with regard to ASFV is strictly limited. So far, only a few papers have reported successful CRISPR/Cas9 targeted ASFV genome modification. The authors were able to get less than $1 \%$ recombinant virus on the initial transfection, suggesting that the method is useful but needs further optimisation to improve its efficiency (4, 13). The other possibility is that CRISPR/Cas9 system performance is simply insufficient to edit all copies of virus genome before ASFV DNA is packaged in its protein envelope during the virus replication cycle in the cell.

Conflict of Interests Statement: The authors declare that there is no conflict of interests regarding the publication of this article.
Financial Disclosure Statement: The conducted study was supported by the project no. UMO2016/21/D/NZ6/00974 entitled "Construction of a recombinant strain of African swine fever (ASFV) using the CRISPR/Cas9 technique and examination of its biological features in vitro and in the pig model" financed by the Polish National Science Centre.

Animal Rights Statement: Not applicable.

\section{References}

1. Abrams C.C., Dixon L.K.: Sequential deletion of genes from the African swine fever virus genome using the cre/loxP recombination system. Virology 2012, 433, 142-148, doi: 10.1016/j.virol.2012.07.021.

2. Barasona J.A., Gallardo C., Cadenas-Fernández E., Jurado C., Rivera B., Rodríguez-Bertos A., Arias M., Sánchez-Vizcaíno J.M.: First oral vaccination of Eurasian wild boar against African swine fever virus genotype II. Front Vet Sci 2019, 6, 1-10, doi: 10.3389/fvets.2019.00137.

3. Borca M.V., Carrillo C., Zsak L., Laegreid W.W., Kutish G.F., Neilan J.G., Burrage T.G., Rock D.L.: Deletion of a CD2-like gene, 8-DR, from African swine fever virus affects viral infection in domestic swine. J Virol 1998, 72, 2881-2889.

4. Borca M.V., Holinka L.G., Berggren K.A., Gladue D.P.: CRISPR-Cas9, a tool to efficiently increase the development of recombinant African swine fever viruses. Sci Rep 2018, 8, 3154, doi: 10.1038/s41598-018-21575-8.

5. Cortiñas Abrahantes J., Gogin A., Richardson J., Gervelmeyer A.: Epidemiological analyses on African swine fever in the Baltic countries and Poland. EFSA J 2017, 15, 4732, doi: 10.2903/j.efsa.2017.4732.

6. de León P., Bustos M.J., Carrascosa A.L.: Laboratory methods to study African swine fever virus. Virus Res 2013, 173, 168-179, doi: 10.1016/j.virusres.2012.09.013.

7. Dixon L.K., Abrams C.C., Bowick G., Goatley L.C., Kay-Jackson P.C., Chapman D., Liverani E., Nix R., Silk R., Zhang F.: African swine fever virus proteins involved in evading host defence systems. Vet Immunol Immunopathol 2004, 100, 117-134, doi: 10.1016/j.vetimm.2004.04.002.

8. Dixon L.K., Sun H., Roberts H.: African swine fever. Antiviral Res 2019, 165, 34-41, doi: 10.1016/j.antiviral.2019.02.018.

9. EFSA AHAW Panel: Scientific opinion on African swine fever. EFSA J 2015, 13, 4163, doi: 10.2903/j.efsa.2015.416.

10. Fernández-Pinero J., Gallardo C., Elizalde M., Robles A., Gómez C., Bishop R., Heath L., Couacy-Hymann E., Fasina F.O., Pelayo V., Soler A., Arias M.: Molecular diagnosis of African swine fever by a new real-time PCR using universal probe library. Transbound Emerg Dis 2013, 60, 48-58, doi: 10.1111/j.1865-1682.2012.01317.

11. Gallardo C., Soler A., Rodze I., Nieto R., Cano-Gómez C., Fernández-Pinero J., Arias M.: Attenuated and nonhaemadsorbing (non-HAD) genotype II African swine fever virus (ASFV) isolated in Europe, Latvia 2017. Transbound Emerg Dis 2019, 66, 1399-1404, doi: 10.1111/tbed.13132.

12. Gómez-Puertas P., Rodríguez F., Oviedo J.M., Brun A., Alonso C., Escribano J.M.: The African swine fever virus proteins p54 and p30 are involved in two distinct steps of virus attachment and both contribute to the antibody-mediated protective immune response. Virology 1998, 243, 461-471, doi: 10.1006/viro.1998.9068.

13. Hübner A., Petersen B., Keil G.M., Niemann H., Mettenleiter T.C., Fuchs W.: Efficient inhibition of African swine fever virus replication by CRISPR/Cas9 targeting of the viral p30 gene (CP204L). Sci Rep 2018, 8, 1449, doi: 10.1038/s41598-018-19626-1. 
14. Jurado C., Martínez-Avilés M., De La Torre A., Štukelj M., de Carvalho Ferreira H.C., Cerioli M., Sánchez-Vizcaíno J.M., Bellini S.: Relevant measures to prevent the spread of African swine fever in the European Union domestic pig sector. Front Vet Sci 2018, 5, doi: 10.3389/fvets.2018.00077.

15. Juszkiewicz M., Walczak M., Woźniakowski G.: Characteristics of selected active substances used in disinfectants and their virucidal activity against ASFV. J Vet Res 2019, 63, 17-25, doi: 10.2478/jvetres-2019-0006.

16. Krug P.W., Holinka L.G., O’Donnell V., Reese B., Sanford B., Fernandez-Sainz I., Gladue D.P., Arzt J., Rodriguez L., Risatti G.R., Borca M.V.: The progressive adaptation of a Georgian isolate of African swine fever virus to Vero cells leads to a gradual attenuation of virulence in swine corresponding to major modifications of the viral genome. J Virol 2015, 89, 2324-2332, doi: 10.1128/JVI.03250-14.

17. Le Rhun A., Escalera-Maurer A., Bratovič M., Charpentier E.: CRISPR-Cas in Streptococcus pyogenes. RNA Biol 2019, 16, 380-389, doi: 10.1080/15476286.2019.1582974.

18. Lee C.: CRISPR/Cas9-based antiviral strategy: current status and the potential challenge. Molecules 201924 (7), 1349, doi: 10.3390/molecules24071349.

19. Lewis T., Zsak L., Burrage T.G., Lu Z., Kutish G.F., Neilan J.G.: An African swine fever virus ERV1-ALR homologue, 9GL, affects virion maturation and viral growth in macrophages and viral virulence in swine. J Virol 2000, 74, 1275-1285, doi: 10.1128/jvi.74.3.1275-1285.2000.

20. Mazur-Panasiuk N., Woźniakowski G., Niemczuk K.: The first complete genomic sequences of African swine fever virus isolated in Poland. Sci Rep 2019, 9, 4556, doi: 10.1038/s41598018-36823-0

21. Monteagudo P.L., Lacasta A., López E., Bosch L., Collado J., Pina-Pedrero S., Correa-Fiz F., Accensi F., Navas M.J., Vidal E., Bustos M.J., Rodríguez J.M., Gallei A., Nikolin V., Salas M.L., Rodríguez F.: BA71 $\triangle \mathrm{CD} 2$ : a new recombinant live attenuated African swine fever virus with cross-protective capabilities. J Virol 2017, 91:e01058-17, doi: 10.1128/JVI.01058-17.

22. Neilan J., Zsak L., Lu Z., Burrage T., Kutish G., Rock D.: Neutralizing antibodies to African swine fever virus proteins p30, p54, and p72 are not sufficient for antibody-mediated protection. Virology 2004, 319, 337-342, doi: 10.1016/j.virol.2003.11.011.

23. O’Donnell V., Holinka L.G., Gladue D.P., Sanford B., Krug P.W., Lu X., Arzt J., Reese B., Carillo C., Risatti G.R., Borca M.V.: African swine fever virus Georgia isolate harboring deletions of MGF360 and MGF505 genes is attenuated in swine and confers protection against challenge with virulent parental virus. J Virol 2015, 89, 6048-6056, doi: 10.1128/JVI.00554-15.

24. O'Donnell V., Holinka L.G., Krug P.W., Gladue D.P., Carlson J., Sanford B., Alfano M., Kramer E., Lu Z., Arzt J., Reese B., Carillo C., Risatti G.R., Borca M.V.: African swine fever virus Georgia 2007 with a deletion of virulence-associated gene 9GL (B119L), when administered at low doses, leads to virus attenuation in swine and induces an effective protection against homologous challenge. J Virol 2015, 89, 8556-8566, doi: 10.1128/JVI.00969-15.

25. O’Donnell V., Risatti G.R., Holinka L.G., Krug P.W., Carlson J., Velazquez-Salinas L., Azzinaro P.A., Gladue D.P., Borca M.V.: Simultaneous deletion of the 9GL and UK genes from the African swine fever virus Georgia 2007 isolate offers increased safety and protection against homologous challenge. J Virol 2017, 91, e01760-1, doi: 10.1128/JVI.01760-16.

26. Oura C.A.L., Denyer M.S., Takamatsu H., Parkhouse R.M.E.: In vivo depletion of $\mathrm{CD} 8+\mathrm{T}$ lymphocytes abrogates protective immunity to African swine fever virus. J Gen Virol 2005, 86, 2445-2450, doi: 10.1099/vir.0.81038-0.

27. Pejsak Z., Truszczyński M., Niemczuk K., Kozak E., Markowska-Daniel I.: Epidemiology of African swine fever in Poland since the detection of the first case. Pol J Vet Sci 2014, 17, 665-672, doi: 10.2478/pjvs-2014-009.

28. Reed L.J., Muench H.: A simple method of estimating fifty per cent endpoints. Am J Epidemiol 1938, 27, 493-497, doi: 10.1093/oxfordjournals.aje.a118408.

29. Reis A.L., Netherton C., Dixon L.K.: Unraveling the armor of a killer: evasion of host defenses by African swine fever virus. J Virol 2017, 91, 6-11, doi: 10.1128/JVI.02338-16.

30. Sánchez E.G., Pérez-Núñez D., Revilla Y.: Development of vaccines against African swine fever virus. Virus Res 2019, 265, 150-155, doi: 10.1016/j.virusres.2019.03.022.

31. Sánchez E.G., Riera E., Nogal M., Gallardo C., Fernández P., Bello-Morales R., López-Guerrero J.A., Chitko-McKown C.G., Richt J.A., Revilla Y.: Phenotyping and susceptibility of established porcine cells lines to African swine fever virus infection and viral production. Sci Rep 2017, 7, 10369, doi: 10.1038/s41598-017-09948-x.

32. Śmietanka K., Woźniakowski G., Kozak E., Niemczuk K., Fraçzyk M., Bocian Ł.: African swine fever epidemic, Poland, 2014-2015. Emerg Infect Dis 2016, 22, 1201-1207, doi: 10.3201/eid2207.151708.

33. Takamatsu H.H., Denyer M.S., Lacasta A., Stirling C.M.A., Argilaguet J.M., Netherton C.L., Oura C.A.L., Nogueira Martins C.E., Rodríguez F.: Cellular immunity in ASFV responses. Virus Res 2013, 173, 110-121, doi: 10.1016/j.virusres.2012.11.009.

34. Woźniakowski G., Kozak E., Kowalczyk A., Łyjak M., Pomorska-Mól M., Niemczuk K.: Current status of African swine fever virus in a population of wild boar in eastern Poland (2014-2015). Arch Virol 2016, 161, 189-195, doi: 10.1007/s00705-015-2650-5.

35. Zani L., Forth J.H., Forth L., Nurmoja I., Leidenberger S., Henke J., Carlson J., Breidenstein C., Viltrop A., Höper D., Sauter-Louis C., Beer M., Blome S.: Deletion at the 5'-end of Estonian ASFV strains associated with an attenuated phenotype. Sci Rep 2018, 8, 6510, doi: 10.1038/s41598-018-24740-1. 